\title{
Exterior elastic fields of non-elliptical inclusions characterized by
}

\section{Laurent polynomials}

\author{
Y.-G. Lee ${ }^{1,2,}{ }^{*}$, W.-N. Zou ${ }^{1, * *}$ \\ ${ }^{1}$ Institute for Advanced Study, Nanchang University, Nanchang 330031, China \\ ${ }^{2}$ Civil Engineering College, Nanchang Institute of Technology, Nanchang 330099, China
}

\begin{abstract}
In this paper, a new method to analytically carry out the exterior elastic fields of a class of non-elliptical inclusions, i.e., those characterized by Laurent polynomials, is developed. Two complex variable fields, which exactly characterize the Eshelby's tensor, are explicitly achieved for the hypocycloidal and the quasi-parallelogram inclusions. Numerical examples show that the exterior fields near the inclusion are dominated by the boundary shape, but the fields far away from the inclusion tend to be convergent and can be well approximated by those of its equivalent circular/elliptical inclusion. These solutions are firstly reported, and largely make up for the deficiency in the list of the analytical results of non-elliptical inclusions in 2D isotropic elasticity.
\end{abstract}

Keywords: Eshelby's tensor; exterior fields; non-elliptical inclusion

\section{Introduction}

Eshelby published his seminal work in two papers (Eshelby, 1957, 1959) about the elastic fields induced by an ellipsoidal inclusion with prescribed uniform eigenstrains in an isotropic infinite body. The first paper reported the most valuable result that the elastic fields are uniform for the interior points, while the second one proposed a closed-form solution for the exterior fields, which was refined and reviewed by Mura (Mura and Cheng, 1977; Mura, 1987). Another two years later, Eshelby (1961) conjectured that, among the inclusions with closed surfaces, the ellipsoidal one alone possesses this remarkable property of uniformity. Ju and Sun (1999) argued that the previous solutions for the exterior field problem are unfinished, and proposed a final and explicit one. Making use of the formulation developed by Ju and Sun (1999), Jin et al. (2011) derived the closed-form solution of the Eshelby tensor for an elliptic cylindrical inclusion where they pointed out that the one given by Kim and Lee (2010) violates the minor symmetry for exterior points. Along with the equivalent inclusion idea becoming a cornerstone

\footnotetext{
* Corresponding authors: yglee@ @it.edu.cn (Y.-G. Lee).

** Corresponding author: zouwn@ncu.edu.cn (W.-N. Zou).
} 
of micromechanics of composites (Mura, 1987; Nemat-Nasser and Hori, 1999; Asaro and Lubarda, 2006), to verify or falsify Eshelby's conjecture became crucial and urgent in many subjects, and brought out a great deal of works, among which various non-ellipsoidal inclusions drew tremendous interest of researchers (Mura et al., 1994; Rodin, 1996; Markenscoff, 1998a, b; Lubarda and Markenscoff, 1998; Ru, 1999; Zou et al., 2010). Physically, the real geometries of inclusions are usually non-ellipsoidal (Onaka et al., 2002a; Onaka, 2008; Miyazawa et al., 2012), which is another impetus of studying non-ellipsoidal inclusion problems.

The boundary of an inclusion could be smooth or flat alone, or a combination of both smooth and flat pieces. Owing to the complexity, the general shapes other than polyhedrons were rarely touched on in three-dimensional (3D) inclusion problems; but for two-dimensional (2D) cases, the representative shapes other than polygons, namely those characterized by Laurent polynomials, were widely tackled (Ru, 1999; Zou et al., 2010). For inclusions with flat boundaries, the analytical solutions of the Eshelby tensor are usually expressed in a summation form over all pieces of the boundary, and are unified for both the interior and exterior points of the inclusion (Rodin, 1996; Nozaki and Taya, 1997; Nozaki and Taya, 2001). However, for inclusions with smooth boundaries, the derivation of the exterior fields must be different from that of the interior ones, and the solutions appear to be more complicated (Eshelby, 1959; Mura, 1987; Ju and Sun, 1999; Ru, 1999). Actually, to the best of the authors' knowledge, limited results are available for the exterior fields of Eshelby's problems when the inclusions involve smooth boundaries. Besides the aforementioned solutions about ellipsoidal and elliptical inclusions, Onaka (2001, 2005; 2002b) treated doughnut-like and superspherical inclusions numerically by the averaged Eshelby's tensor over the inclusion; Ru (1999), making use of complex elastic potentials and the techniques of analytical continuation and conformal mapping, developed a general method and obtained analytical solution for a 2D arbitrarily shaped inclusion, and yet explicitly presented the exterior fields of an elliptical inclusion alone; Zou et al. (2010) derived new boundary integral expressions associated with the Eshelby's tensor fields in 2D elasticity, and explicitly achieved the expressions of the interior Eshelby tensor fields and their averages for various non-elliptical inclusions characterized by Laurent polynomials, but did not provided the corresponding exterior Eshelby's tensor fields.

As Eshelby (1959) pointed out that "the elastic field inside the inclusion can be calculated without having to find the field outside the inclusion", and that "a good deal of information can be derived from a knowledge of the internal field alone", the exterior fields are at least significant when concerning: (1) the integrity of Eshelby's tensor fields, (2) the interaction between inclusions, (3) the influence range upon the matrix, and (4) the interaction between inclusions and finite boundaries, defects, etc.. In view of these and the aforementioned points, 
in this paper, we proposed a novel formulation to obtain the analytical expressions of the exterior elastic fields of inclusions described by Laurent polynomials.

The content of this paper is as follows. In Section 2, we recall the basic results of the boundary integral expressions of the Eshelby's tensor fields developed by Zou et al. (2010). In Section 3, we formulate the general solution of the exterior Eshelby's tensor fields for inclusions characterized by Laurent polynomials. In Section 4, explicit results of the hypocycloidal and the quasi-parallelogram inclusions are presented and illustrated, and the influence range of the exterior fields is discussed. In the last section, we make some concluding remarks. In Appendix A, we list the explicit results of the interior Eshelby's tensor fields for the inclusions discussed in Section 4. The solutions of arbitrary polygonal inclusions are presented in Appendix B.

\section{Boundary integral expressions of Eshelby's tensor fields}

In this section, following Zou et al. (2010), we present the boundary integral results of Eshelby's tensor fields in 2D spaces. Let $w$ be an inclusion of an isotropic unbounded body and undergo a uniform eigenstrain $\left\{e_{i j}^{*}\right\}$. Then, the perturbed stress field $\left\{s_{i j}\right\}$ can be expressed in terms of $\left\{e_{i j}^{*}\right\}$, i.e.,

$s_{i j}=C_{i j k l}\left(\mathrm{~S}_{k l m n}^{w}-I_{k l m n} c^{w}\right) e_{m n}^{*}$,

where $\left\{\mathrm{S}_{k l m n}^{w}\right\}$ is the Eshelby's tensor, relating the total strain $\left\{e_{k l}\right\}$ and the prescribed eigenstrain $\left\{e_{m n}^{*}\right\}$ by

$e_{k l}=\mathrm{S}_{k l m n}^{w} e_{m n}^{*}$.

In (1), $\left\{C_{i j k l}\right\}$ is the stiffness tensor of the material, $I_{i j k l}=\frac{1}{2}\left(d_{i k} d_{j l}+d_{i l} d_{j k}\right)$ is the components of the fourth-order unit tensor, $c^{w}$ is the characteristic function of the inclusion $w$ that equals to one or zero according to the field point $\mathbf{x}$ locating inside or outside of $w$. Throughout this paper, the components of a tensor are measured in a fixed rectangular Cartesian coordinate system and the Einstein summation convention is adopted, i.e., repeated indices in each term mean the summation over 1 and 2.

From Zheng et al. (2006), the following irreducible decomposition 
$\mathrm{S}_{i j k l}^{w}(\mathbf{x})=\mathrm{S}_{i j k l}^{o} c^{w}+A_{i j k l}^{w}(\mathbf{x})$

is available for the Eshelby's tensor associated with an inclusion embedded in a 2D infinite medium, where the isotropic part $\left\{\mathrm{S}_{i j k l}^{o}\right\}$ and the anisotropic part $\left\{A_{i j k l}^{w}(\mathbf{x})\right\}$ are indicated by

$\mathrm{S}_{i j k l}^{o}=\frac{2-k}{2(k+1)} d_{i j} d_{k l}+\frac{k}{2(k+1)}\left(d_{i k} d_{j l}+d_{i l} d_{j k}\right), A_{i j k l}^{w}=\frac{k-1}{k+1} d_{i j} d_{k l}^{w}+\frac{2}{k+1} d_{k l} d_{i j}^{w}+\frac{4}{k+1} D_{i j k l}^{w}$,

respectively, with $d_{i j}$ being the components of the Kronecker delta, $\left\{d_{i j}^{w}\right\}$ and $\left\{D_{i j k l}^{w}\right\}$ the second-order and fourth-order deviators, respectively, and

$k=\frac{3-n}{1+n}$, plane stress;

The deviators $\left\{d_{i j}^{w}\right\}$ and $\left\{D_{i j k l}^{w}\right\}$ are material-independent, and can be expressed in terms of integrals over the inclusion domain, i.e.,

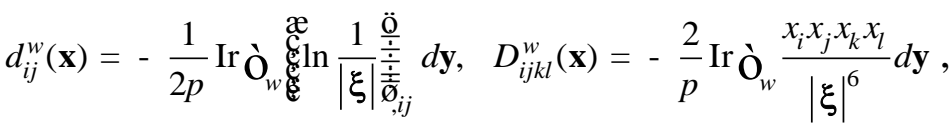

where 'Ir' denotes taking the traceless-symmetric part of a tensor, $\xi=\mathbf{y}-\mathbf{x}, \quad()_{, i}$ designates partial derivative with respect to variable $x_{i}$, the boldface letters $\mathbf{x}$ and $\mathbf{y}$ denote the position vectors of the field point $\left(x_{1}, x_{2}\right)$ and the source point $\left(y_{1}, y_{2}\right)$.

Since an arbitrary 2D deviator has up to two independent components, denoting by

$p_{2}=d_{11}^{w}, q_{2}=d_{12}^{w} ; p_{4}=D_{1111}^{w}$, and $q_{4}=D_{1112}^{w}$,

we are able to use two complex variables

$\mathrm{G}_{2}=\& p_{2}+i q_{2}, \mathrm{G}_{4}=\& p_{4}+i q_{4}$

to provide a full view of the components of $d_{i j}^{w}$ and $D_{i j k l}^{w}$, wherein $i=\sqrt{-1}$ is the unit imaginary number. Substituting (6) and (7) into (8), and making use of the representation of traceless-symmetric tensors and the Green integral theorem, we reach the following boundary integral expressions of $\Gamma_{2}$ and $\Gamma_{4}$

$2 \overline{\mathrm{G}}_{2}=-\frac{1}{2 p i} \tilde{\mathbf{\Phi}_{w}} \frac{d \bar{y}}{x}, 8 \overline{\mathrm{G}}_{4}=-\frac{1}{2 p i} \tilde{\mathbb{N}_{w}} \frac{\bar{x}}{x^{2}} d \bar{y}$, 
where $\overline{(y}$ denotes the complex conjugate of a variable, $x=x_{1}+i x_{2}, y=y_{1}+i y_{2}$ and $x=y-x$ are the complex representations of the position vectors $\mathbf{x}, \mathbf{y}$ and the relative position vector $\xi=\mathbf{y}-\mathbf{x}$, respectively. It should be noted that in (9) we presents the conjugates, rather than $\Gamma_{2}$ and $\Gamma_{4}$ themselves, to offer convenience in later derivations. We also remark that the boundary integral expressions in (9) are applicable for both the interior and exterior field points of the inclusion. The stress and induced strain fields can be achieved through (1) and (2).

For easy use, we introduce the matrix form of Eshelby's tensor field, i.e.,

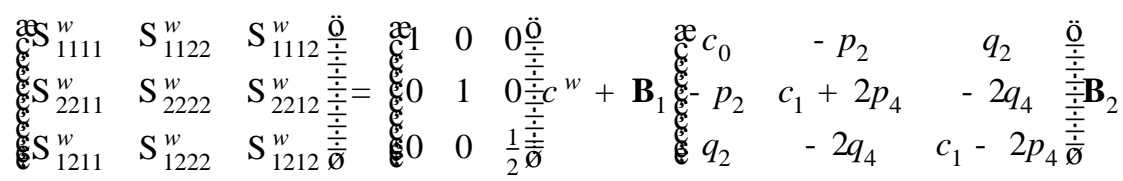

where $c_{0}=-\frac{1}{2} c^{w}, c_{1}=-\frac{1}{4} c^{w}$,

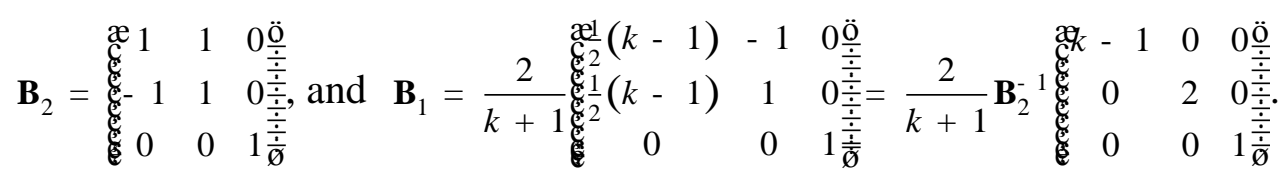

The above compact expression was first proposed by Zou et al. (2012), however, it should be pointed out that a few typos happened in the expression of $\mathbf{B}_{1}$ in Eq. (69) of the paper.

In summary, solutions of the inclusion problem in a 2D infinite isotropic medium are attributed to calculating two governing functions $\Gamma_{2}$ and $\Gamma_{4}$ which involve only the boundary integrals of the inclusion domain. The formulae (10) and (11) further show that $\Gamma_{2}$ and $\Gamma_{4}$ characterize the Eshelby's tensor to the best advantage. In this sense, we will put our focus on the calculation and discussion of $\Gamma_{2}$ and $\Gamma_{4}$ in the following sections.

\section{General solution of the exterior fields for inclusions}

\section{characterized by Laurent polynomials}

The Laurent polynomial (Henrici, 1986; Zou et al., 2010)

$$
x=x_{0}+R\left(w+\AA_{k=1}^{N} b_{k} w^{-k}\right),|w|^{3} 1, x_{0} \hat{I} \quad w, R>0,
$$


gives a one-to-one conformal mapping from the exterior of the unit disk with the origin as its center to the exterior of the inclusion with the origin as its interior point. By taking $|w|=1$, Eq. (12) also characterizes the simply closed boundary of the inclusion which is mapped from a unit cycle. The above mapping relation must admit the following factorization

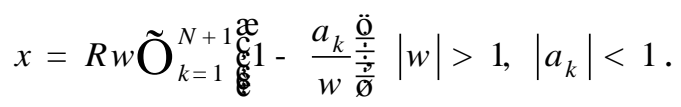

Now, we consider the Jordan curve $\uparrow \mathrm{W}_{x}$ defined by

$$
x=x_{0}+R\left(w+\stackrel{\circ}{N}{ }_{k=1}^{N} b_{k} w^{-k}\right),|w|=\text { constant }>1 .
$$

It is obvious that the points defined by $h$, instead of $w$ in (12), with $|h|=1$ must be the interior points of $\uparrow \mathrm{W}_{x}$, which implies that the factorization

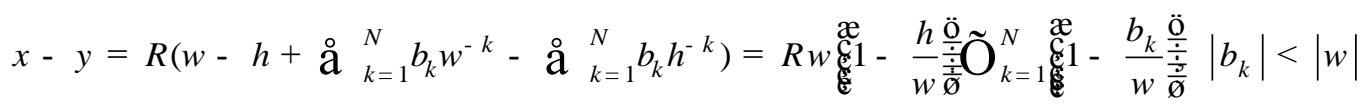

holds, where

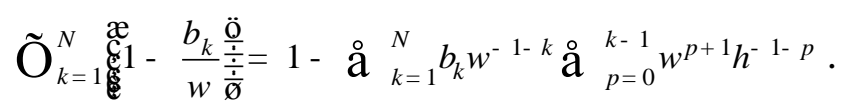

The above property can be used to carry out the expansions

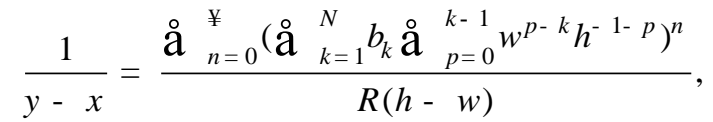

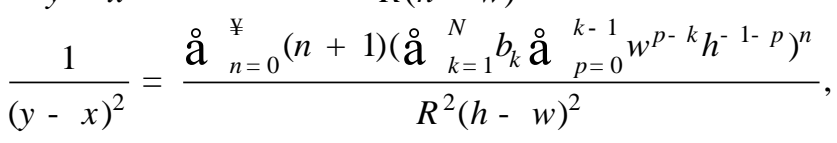

where $y$ and $x$ indicate points on the boundary of the inclusion and in the exterior of the inclusion, respectively. By virtue of (17), the integrals in (9) can be explicitly worked out, i.e.,

$$
\begin{gathered}
2 \overline{\mathrm{G}}_{2}=-\frac{1}{2 p i} \tilde{\mathbf{A}}_{w} \frac{d \bar{y}}{y-x}=-\frac{1}{2 p i} \tilde{\mathbf{W}}_{h=1} \frac{G_{2}(h, w) d h}{h-w}=G_{2}(w, w)-D_{2}(w, w), \\
8 \overline{\mathrm{G}}_{4}=-\frac{1}{2 p i} \tilde{\mathbf{W}_{w}} \frac{\bar{y}-\bar{x}}{(y-x)^{2}} d \bar{y}=-\frac{1}{2 p i} \tilde{\mathbf{W}}_{h \mid=1} \frac{G_{4}(h, w) d h}{(h-w)^{2}}=G_{4}^{\prime}(w, w)-D_{4}^{\prime}(w, w),
\end{gathered}
$$

where

$$
G_{2}(h, w)=D_{2}(h, w)+o\left(h^{-1}\right), G_{4}(h, w)=D_{4}(h, w)+o\left(h^{-1}\right), \text { as }|h| \stackrel{\circledR}{\circledR} .
$$


In Eqs. (18) and (19), $G_{2}(h, w)$ and $G_{4}(h, w)$ are polynomials of variable $h$, whilst $D_{2}(h, w)$ and $D_{4}(h, w)$ extract only the terms of positive powers from $G_{2}(h, w)$ and $G_{4}(h, w)$, respectively. It should be mentioned that $G_{4}{ }^{\prime}(w, w)$ and $D_{4}^{\prime}(w, w)$ in (18) mean

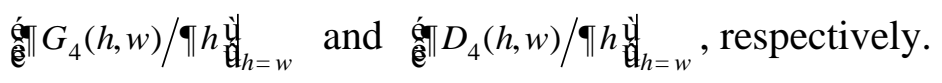

Eq. (18) infers that $\Gamma_{2}$ and $\Gamma_{4}$ can be explicitly expressed in terms of basic functions of variable $w$, besides a linear relation with respect to $\bar{x}$. Further, the induced strain and stress fields can be achieved in a straight forward manner. In practice of calculations, $w$, associated with $x$, can be determined as the maximal-module root of the polynomial equation of $w$

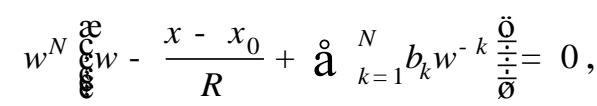

which is deformed from the conformal mapping (12). The beginning point of explanation is that the mapping (12) with factorization (13) provides a one-to-one correspondence from the exterior of the unit disk to the exterior of the inclusion. Then, based on this viewpoint, for the exterior point $x$ in the physics plane given by an exterior point, say $w_{0}\left(\left|w_{0}\right|=a^{3} 1\right)$, in the image plane, the root of (20) having maximal module larger than $a$ is impossible, and $w_{0}$ must be the unique root with module $a$; Otherwise, there are more than one exterior points in the image plane corresponding to the exterior point $x$ in the physics plane.

\section{Explicit results, illustrations and discussions}

In this section, following the general solution procedure shown in Section 3 and considering the hypocycloidal inclusion and the quasi-parallelogram one, we explicitly derived the expressions of $\mathrm{G}_{2}$ and $\mathrm{G}_{4}$ for the exterior points of the inclusion. Then, after specifying the geometric parameters of the inclusion, we illustrate the exterior fields together with the corresponding interior ones for integrity. For comparison, the fields of a circular or an elliptical inclusion and those of regular polygonal inclusions with similar geometric features are also figured out. Finally, the influence ranges of the exterior fields are discussed in detail. As complements, the explicit expressions of $\mathrm{G}_{2}$ and $\mathrm{G}_{4}$ for corresponding interior points and those for arbitrary polygonal inclusions are pasted as Appendices A and B, which are taken from Zou et al. (2010). 


\subsection{Hypocycloidal inclusions}

The mapping

$y=h+b_{N} h^{-N},|h|=1, N^{3} 1$

gives rise to the hypocycloidal inclusions: an ellipse for $N=1$, a regular curvilinear $(N+1)$-polygon for $N>1$ (Ru, 1999; Zou et al., 2010). Starting from this shaped inclusion, the explicit expressions of $\Gamma_{2}$ and $\Gamma_{4}$ can be derived as

$$
\begin{aligned}
2 \overline{\mathrm{G}}_{2} & =-\frac{w^{-1}-N \bar{b}_{N} w^{N}}{w-N b_{N} w^{-N}}-N \bar{b}_{N} x^{N-1}, \\
8 \overline{\mathrm{G}}_{4} & =\frac{w^{-1}+\bar{b}_{N} w^{N}-\bar{x}}{\left(w-N b_{N} w^{-N}\right)^{3}} \dot{e}^{N}(N-1) \bar{b}_{N} w^{N+1}+N(N-1) b_{N} w^{-N-1}-2 N^{3} b_{N} \bar{b}_{N}+2 \text { 狊 } \\
& +\frac{\left(w^{-1}-N \bar{b}_{N} w^{N}\right)^{2}}{\left(w-N b_{N} w^{-N}\right)^{2}}+N(N-1) \bar{b}_{N} \bar{x} x^{N-2}-(N-1)(N-2) \bar{b}_{N}\left(1-N b_{N} \bar{b}_{N}\right) x^{N-3}-N(2 N-1) \bar{b}_{N}^{2} x^{2 N-2} .
\end{aligned}
$$

The above formulae are unified for any natural number $N$. As for the ellipse with $N=1$, the solution is further simplified as

$$
2 \overline{\mathrm{G}}_{2}=-\frac{1-b_{1} \bar{b}_{1}}{w-b_{1} w^{-1}} w^{-1}, 8 \overline{\mathrm{G}}_{4}=\left(1-b_{1} \bar{b}_{1}\right) \frac{\left(1+b_{1} \bar{b}_{1}\right)\left(3 w^{-1}-b_{1} w^{-3}\right)-2 \bar{x}}{\left(w-b_{1} w^{-1}\right)^{3}},
$$

which can be verified to be consistent with the known results ( $\mathrm{Ru}, 1999$; Jin et al., 2011).

In the following, the deltoid $(N=2)$ and astroid $(N=3)$ inclusions are taken for example to show their Eshelby's tensor fields $\Gamma_{2}$ and $\Gamma_{4}$. As comparison, the corresponding regular polygonal inclusions are illustrated together. In order to carry out the comparison of different inclusions, the area equivalence idea is used, namely the coordinate measures for different inclusions should be changed to keep all inclusions having the same area when their Eshelby's fields are compared point-by-point. In practice, we normalize every shape by a unit circle. For the inclusion $w$ characterized by the Laurent polynomial

$y=h+\stackrel{\mathrm{a}}{N}{ }_{k=1}^{N} b_{k} h^{-k},|h|=1$,

its area can be expressed as (Zou et al., 2010)

$|w|=\grave{\mathbf{O}}_{w} d A=\frac{1}{2 i} \tilde{\mathbf{A}}_{w} \bar{y} d y=p\left(1-\stackrel{\mathrm{a}}{k=1}_{k=1}^{N} k b_{k} \bar{b}_{k}\right)$,

and its normalized factor $R$ (as shown in (12)) should be calculated by 
$R=\sqrt{p /|w|}=\sqrt{\left(1-\stackrel{\mathrm{a}}{k=1}_{k=1}^{N} k b_{k} \bar{b}_{k}\right)^{-1}}$.

In Fig. 1, the fields of $\Gamma_{2}$ and $\Gamma_{4}$ for two deltoid inclusions $\left(b_{2}=\frac{1}{2}\right.$ for a concave shape and $b_{2}=\frac{1}{6}$ for a convex one) are plotted together with those of a regular triangular one. As expected, the exterior fields of the three inclusions share the same feature far from the inclusions, which can be shown in Fig. 1. It is further shown that the real parts of $\Gamma_{2}$ and $\Gamma_{4}$ both within and outside of the inclusion have reflection symmetry with respect of the $x_{1}$-axis while the image parts are anti-reflection symmetric in the same manner. The left two columns in Fig. 1 show that the real parts of $\Gamma_{2}$ within the inclusions exhibit nearly uniform gradients (but different between the three inclusions) along the $x_{1}$-axis while the image parts exhibit similar feature along the $x_{2}$-axis. The right two columns in Fig. 1 show that the fields of $\Gamma_{4}$ within the inclusions exhibit rather non-uniform gradients.

(a)

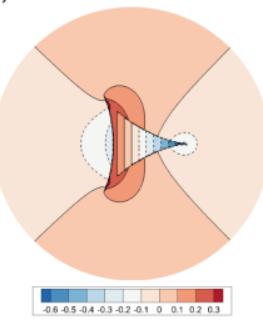

(e)

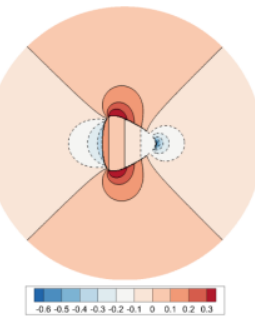

(1)

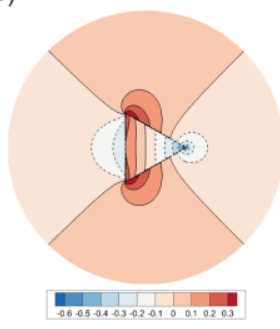

(b)

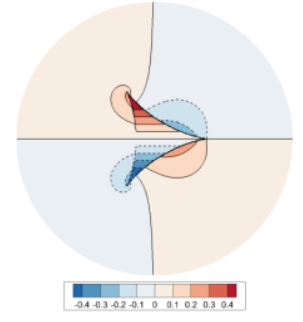

(f)

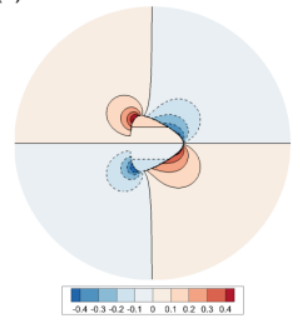

(j)

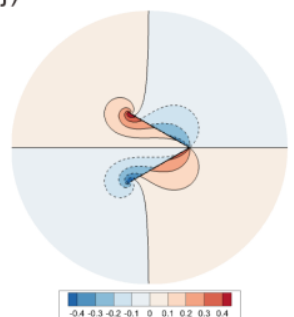

(c)

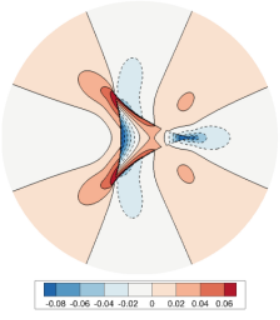

(g)

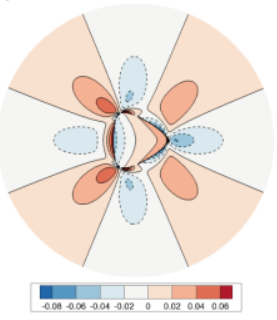

(k)

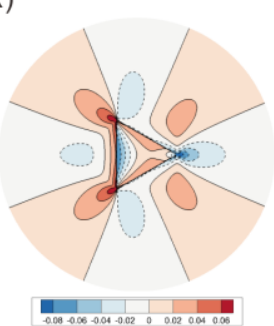

(d)

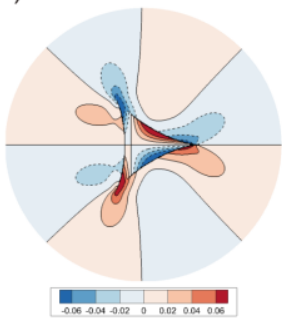

(h)

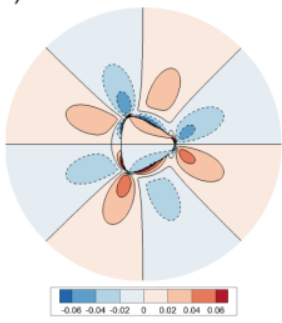

(I)

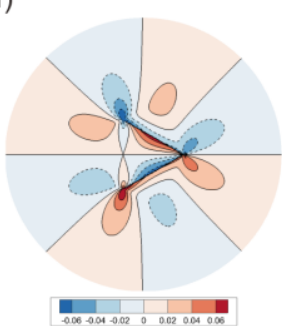

Fig. 1. Normalized $G_{2}$ and $G_{4}$ of two deltoid (3-gonal hypocycloid) inclusions and a regular triangular one.

Rows from top to bottom show the fields corresponding to deltoids with $b_{2}=\frac{1}{2}, b_{2}=\frac{1}{6}$ and regular triangle with side length $\sqrt{3}$; Columns from left to right correspond to the fields of $\operatorname{Re} G_{2}, \operatorname{Im} G_{2}, \operatorname{Re} G_{4}$ 
and $\mathrm{Im} \mathrm{G}_{4}$. The normalized factors of the three shapes are $\sqrt{2}=1.414, \sqrt{\frac{18}{17}}=1.029$ and $\sqrt{\frac{4 p}{3 \sqrt{3}}}=1.555$, respectively.

Subsequently, an astroid inclusion with $b_{3}=-\frac{1}{4}$, a normal square one with side length 2 and a unit circular one are considered concurrently, as shown in Fig. 2. It can be seen that the exterior fields of $\mathrm{G}_{2}$ are equally divided into four sections by zero-valued contour lines while those of $\mathrm{G}_{4}$ are partitioned into eight. The real parts of $\mathrm{G}_{2}$ and $\mathrm{G}_{4}$ have reflection symmetry with respect to both the $x_{1}$ and $x_{2}$ axes while the image parts of them exhibit anti-reflection symmetry the same manner. As expected, with respect to the $\pm \frac{\pi}{4}$ lines, the real parts of $\mathrm{G}_{2}$ and $\mathrm{G}_{4}$ are anti-reflection symmetric while their image parts are reflection symmetric. It is remarkable that the aforementioned features are shared by the three cases of inclusion. However, the interior fields of the three cases exhibit much difference from which it can be roughly concluded that the concavity of the curvilinear boundary generates rather non-uniform interior fields but exerts little influence on the exterior fields.

(a)

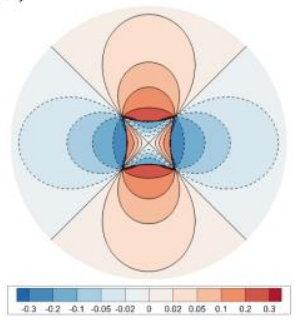

(e)

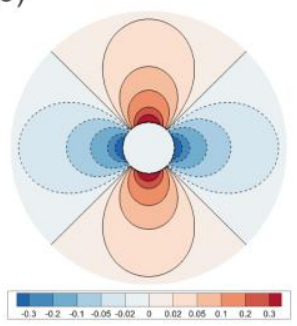

(I)

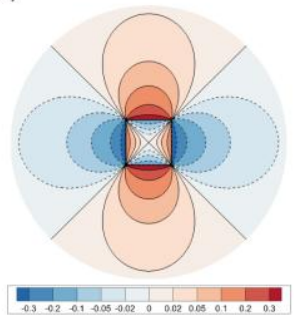

(b)

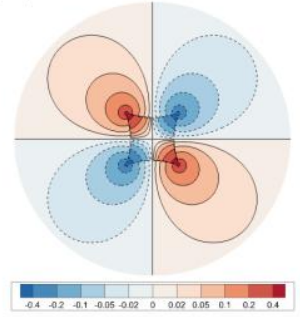

(f)

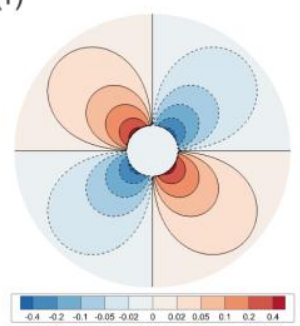

(j)

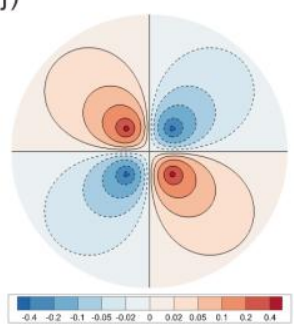

(c)

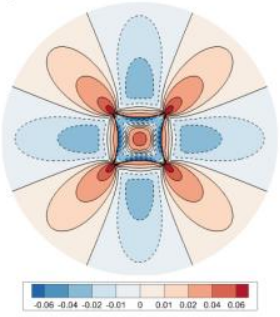

(g)

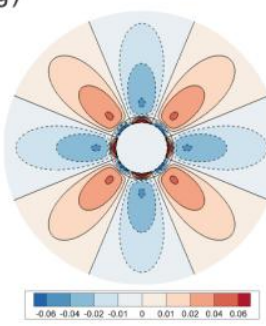

(k)

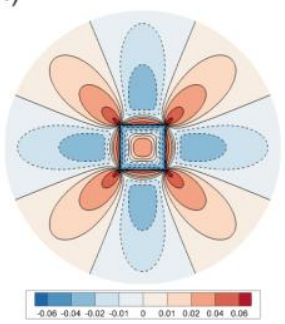

(d)

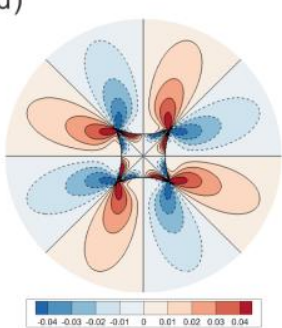

(h)

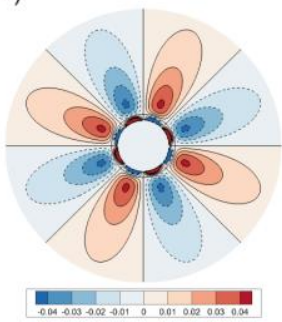

(I)

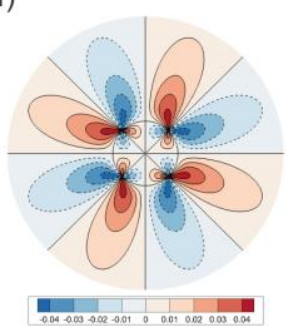

Fig. 2. Normalized $\mathrm{G}_{2}$ and $\mathrm{G}_{4}$ of an astroid (4-gonal hypocycloid) inclusion with $b_{3}=-\frac{1}{4}$, a unit circular inclusion and a normal square one with side length 2. Rows from top to bottom show the fields of the astroid, the circular and the square inclusions; Columns from left to right correspond to the fields of 
$\operatorname{ReG}_{2}, \operatorname{Im} G_{2}, \operatorname{ReG}_{4}$ and $\operatorname{ImG}_{4}$. The normalized factors of the three shapes are $\frac{4}{\sqrt{13}}=0.901,1$ and $\frac{\sqrt{p}}{2}=0.886$, respectively.

\subsection{Quasi-parallelogram inclusions}

In this subsection, we consider the quasi-parallelogram inclusion described by $y=h+b_{1} h^{-1}+b_{3} h^{-3}$. From (18), the explicit expressions of $\mathrm{G}_{2}$ and $\mathrm{G}_{4}$ for the exterior points can be derived as

$$
\begin{aligned}
& 2 \overline{\mathrm{G}}_{2}=-\frac{w^{-1}-\overline{b_{1}} w-3 \bar{b}_{3} w^{3}}{w-b_{1} w^{-1}-3 b_{3} w^{-3}}-\left(\bar{b}_{1}-3 b_{1} \bar{b}_{3}\right)-3 \bar{b}_{3} x^{2}, \\
& 8 \overline{\mathrm{G}}_{4}=8 b_{1} \overline{b_{1}} \bar{b}_{3}-\left(2 \bar{b}_{3}+\bar{b}_{1}^{2}\right)+3 \bar{b}_{3}^{2}\left(2 b_{3}-3 b_{1}^{2}\right)+6 \bar{b}_{3} \bar{x} x+12 \bar{b}_{3}\left(3 b_{1} \bar{b}_{3}-\bar{b}_{1}\right) x^{2}-15 \bar{b}_{3}^{2} x^{4} \\
& \quad+\frac{2\left(w^{-1}+\bar{b}_{1} w+\bar{b}_{3} w^{3}-\bar{x}\right)\left[1-b_{1} \bar{b}_{1}-27 b_{3} \bar{b}_{3}+3 \bar{b}_{3}\left(w^{4}-2 b_{1} w^{2}\right)+3 b_{3}\left(w^{-4}-2 \bar{b}_{1} w^{-2}\right)\right]}{\left(w-b_{1} w^{-1}-3 b_{3} w^{-3}\right)^{3}}+\frac{\left(w^{-1}-\bar{b}_{1} w-3 \bar{b}_{3} w^{3}\right)^{2}}{\left(w-b_{1} w^{-1}-3 b_{3} w^{-3}\right)^{2}} .
\end{aligned}
$$

Then, we specify the geometry of the quasi-parallelogram by $b_{1}=\frac{1}{2}$ and $b_{3}=-\frac{1}{4}$, and plot the fields of $\mathrm{G}_{2}$ and $\mathrm{G}_{4}$ in the first row of Fig. 3, where the interior fields are taken from (A.3). In Fig. 3, the fields of a rectangular inclusion with its center at the origin, length 5 and height 1 , and those of an origin-centered elliptical inclusion with semi-major axis $\frac{5}{3}$ and semi-minor axis $\frac{1}{3}$ are also illustrated as comparison. The symmetry properties with respect to the $x_{1}$ and $x_{2}$ axes showed in Fig. 3 are completely the same as those in Fig. 2. However, the symmetry properties with respect to the $\pm \frac{p}{4}$ inclined lines are lost. The bottom row of Fig. 3 shows that the real parts of $G_{2}$ and $G_{4}$ are discontinuous across the boundary of the rectangular inclusion while the image parts of them are continuous. The interior fields of the three inclusions illustrate distinct features: the fields of the quasi-parallelogram inclusion and the rectangular inclusion are quite non-uniform, especially near the boundary corners, while those for the elliptical inclusion are uniform.

By now, the exterior field solutions of two kinds of inclusion are explicitly presented and illustrated. For each kind of inclusion, cases of concave and convex shapes are tried to put together for comparison, along with the same featured polygons. It is mentioned that the concavity or convexity of the inclusion shape depends on the geometric parameters $\left(b_{1}, b_{2}, b_{3}\right.$, etc.), but the detailed discussions are beyond the scope of this paper. Readers who are interested in this issue can refer to the monograph by Muskhelishvili (1977) for more information. 
(a)

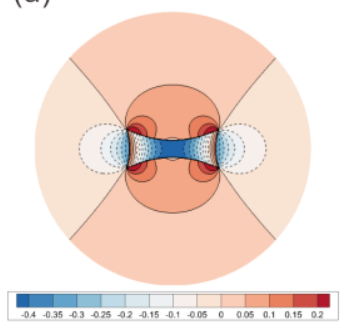

(e)

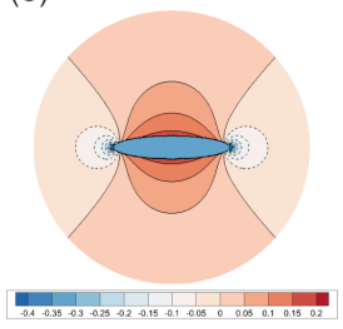

(I)

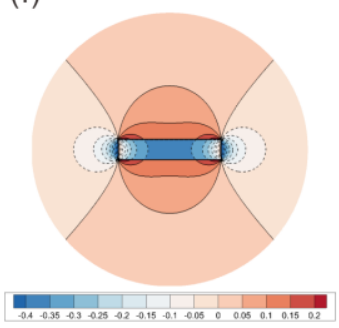

(b)

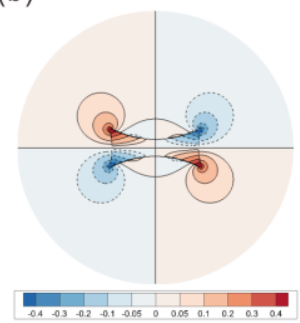

(f)

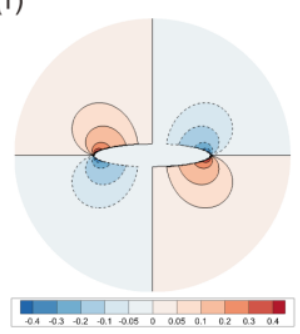

(j)

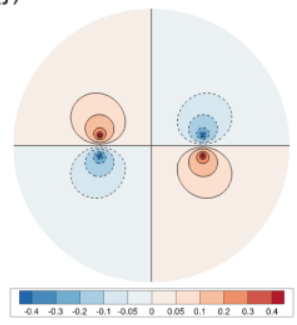

(c)

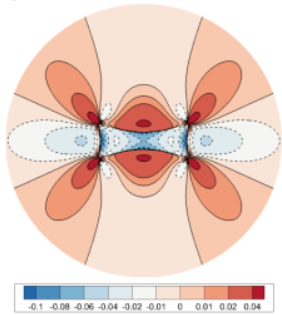

(g)

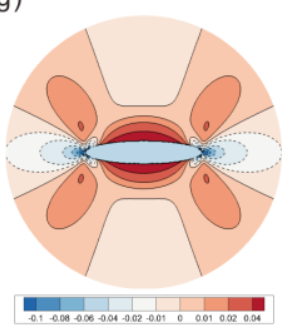

(k)

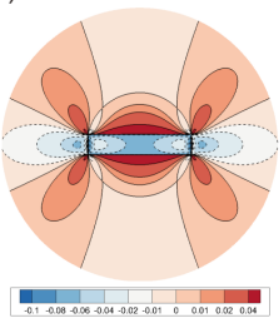

(d)

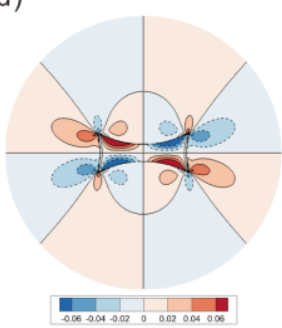

(h)

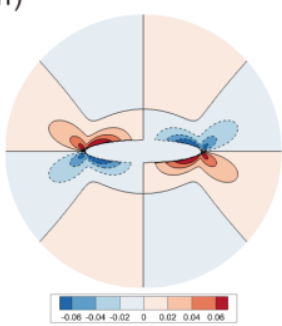

(l)

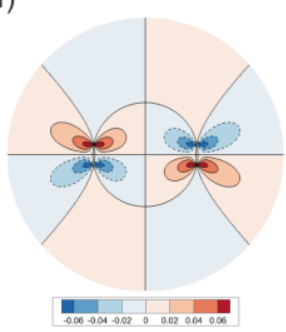

Fig. 3. Normalized $\mathrm{G}_{2}$ and $\mathrm{G}_{4}$ of a quasi-parallelogram inclusion with $b_{1}=\frac{1}{2}$ and $b_{3}=-\frac{1}{4}$, an elliptical inclusion with semi-major axis $\frac{5}{3}$ and semi-minor axis $\frac{1}{3}$, and a rectangular inclusion with length 5 and height 1 . Rows from top to bottom show the fields of the quasi-parallelogram, the elliptical and the rectangular inclusion, respectively. Columns from left to right correspond to the fields of $\operatorname{Re}_{2}, \quad I m G_{2}, \quad R e G_{4}$ and $\operatorname{Im~G}_{4}$. The normalized factors of the three shapes are $\frac{4}{3}=1.333, \frac{3}{\sqrt{5}}=1.342$ and $\sqrt{\frac{p}{5}}=0.793$, respectively.

\subsection{Influence range of the exterior fields}

The solutions of circular and elliptical inclusions are widely used in all kinds of estimate methods of micromechanics, partly due to the equivalent inclusion method (EIM) based on the Eshelby's uniformity property of elliptical inclusions, and partly originating from one's subconsciousness that the effect of an arbitrary inclusion could be replaced by that of a circular or elliptical inclusion to some extent. When the solutions of various non-elliptical inclusions become available, some solid analyses can be carried out to investigate the influence of different shapes in the far field and consequently the validity of circle or ellipse approximations. 
Because the far fields of $\mathrm{G}_{2}$ and $\mathrm{G}_{4}$ for the circular/elliptical inclusion are nonzero except for the infinity, we are capable of defining the relative errors of exterior fields $\mathrm{G}_{2}$ and $\mathrm{G}_{4}$ of an inclusion along a direction $q(q=\arg (x)$ for field point $x)$

$\mathrm{D}_{q, p}^{* \cdot}=\left|\mathrm{G}_{p}^{*}-\mathrm{G}_{p} \cdot\right| /\left|\mathrm{G}_{p}\right|, p=2,4$

with respect to its equivalent circular/elliptical inclusion, where the superscript asterisk '*' and black point ' $\bullet$ ' stand for the specified inclusion and the associated circular/elliptical inclusion $(\bullet=c$ for circle and $e$ for ellipse), respectively. The equivalent circular/elliptical inclusion of an inclusion means that they have (1) the same center position, (2) the same inclusion area, and (3) the same ratio and direction of principal axis of inertia for the elliptical inclusion. We further specify threshold values $5 \%$ and $1 \%$ of $\mathrm{D}_{q, p}^{*, \cdot}$ as the criteria of rough and good approximation, respectively.

For simplicity, only shapes with two orthogonal symmetric axes are considered, such as a circle, an ellipse, a square, a rectangle, an astroid, and a quasi-parallelogram, etc. As shown in Fig. 2 and Fig. 3, the exterior fields near the inclusion are quite distinct for different inclusions. However, as the distance between the field point and the inclusion's center increases, the exterior fields of all the inclusions reduce rapidly and tend to converge, first to those of an elliptical inclusion and further to those of a circular inclusion. In order to make a reasonable comparison between different inclusions, we rescale the coordinate by the inclusion's normalized factor, as shown in Figs. 1-3, or Table 1. Since the remote properties with the orientation are the same, we are concerned with the modules of $G_{2}$ and $G_{4}$ or the extreme values of their components. Due to the symmetry features, $\operatorname{Im~}_{2}$ and $\operatorname{Im~}_{4}$ always vanish along the coordinate axes (see Fig. 2 and Fig. 3), so it is comprehensive to inspect $\operatorname{Re}_{2}$ and $\mathrm{Re}_{4}$ along the coordinate axes for all involved inclusions.

We first consider a square and an astroid inclusion, and study the variation of $\operatorname{ReG}_{2}$ and $\mathrm{ReG}_{4}$ along the $x_{1}$-axis, by comparing them with the equivalent circular inclusion. The results are plotted in Fig. 4. And in the upper part of Table 1, we list the positions and the field values at the good and rough thresholds when the inclusions are approximated by their equivalent circular ones. Fig. 4 shows that $\mathrm{ReG}_{2}$ and $\mathrm{ReG}_{4}$ jump across the interface between the inclusion and the matrix along the $x_{1}$-axis for all the cases. It can be concluded from Table 1 
that for a circle-type inclusion, the exterior fields can be well approximated by those of a circular inclusion when the distance from the center exceeds 4 under a relative error 5\%, and 6 under a relative error $1 \%$, where the corresponding area fractions are about $6 \%$ and $3 \%$, respectively.

Then, we consider a rectangular and a quasi-parallelogram inclusion in comparison with an elliptical one, and study the variations of $\mathrm{ReG}_{2}$ and $\mathrm{ReG}_{4}$ along both coordinate (principal) axes. The three inclusions share the same center, the same area and the same major-minor axis ratio 5. The variation trends are shown in Fig. 5. In the lower part of Table 1, we list the positions and the field values at the good and rough thresholds when the inclusions are approximated by their equivalent elliptical and circular ones. According to Table 1, we can conclude that, in the scale of equivalent circle: (1) The exterior fields approach those of an elliptical inclusion when the distances from the center exceed 5 under a relative error $5 \%$, and 9 under a relative error $1 \%$, of which the corresponding area fractions are about $4 \%$ and $1.2 \%$, respectively. If measured by the maximal (major axis) length (2.23 in the case of ellipse), the positions will be about half close to the center. (2) As for the circle approximation, the distances change to 15 under a relative error $5 \%$, and 32 under a relative error $1 \%$, and the corresponding area fractions become rather small.

(a)

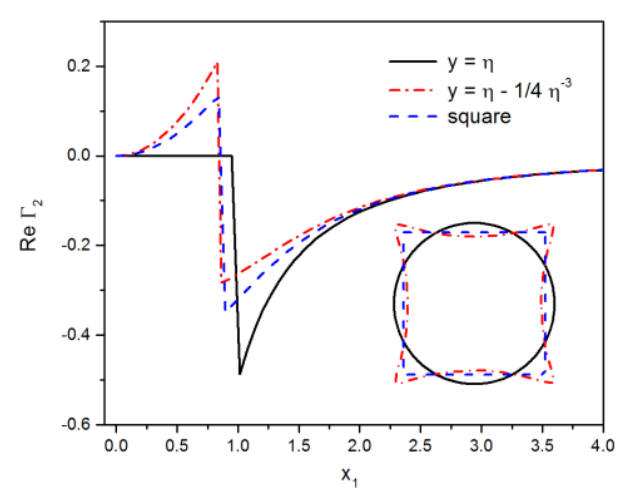

(b)

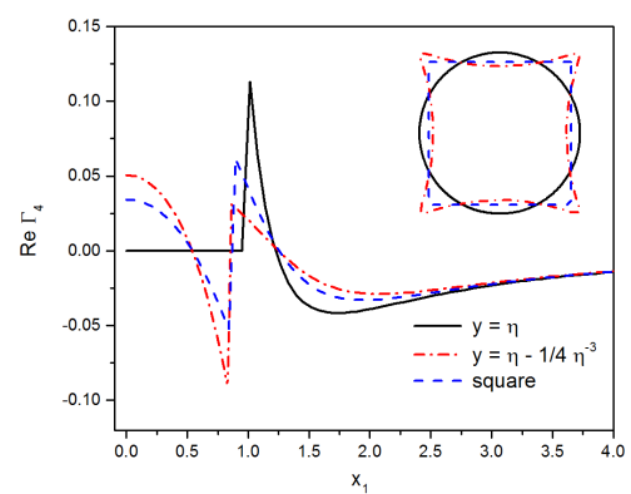

Fig. 4. Variations of $\operatorname{ReG}_{2}$ and $\mathrm{ReG}_{4}$ along the $x_{1}$-axis for the three inclusions: circular, astroid, and square, respectively. The inserted figures in the right corners mark the rescaled boundaries of the inclusions. 
Table 1. The influence ranges of different shapes of inclusions when approximated by an elliptical or a circular inclusion. (Shape codes: Q - Quasi-parallelogram, R - Rectangle, A - Astroid, S - Square). The number in the parentheses next to the shape name (code) indicates the normalized factor of the inclusion.

\begin{tabular}{|c|c|c|c|c|c|c|c|c|c|c|}
\hline & & & \multicolumn{4}{|c|}{ Ellipse (1.342) approximation range } & \multicolumn{4}{|c|}{ Circle (1) approximation range } \\
\hline & & & \multicolumn{2}{|c|}{$\operatorname{Rough}\left(\mathrm{D}_{q, p}^{*, e} ; 5 \%\right)$} & \multicolumn{2}{|c|}{ Good $\left(\mathrm{D}_{q, p}^{*, e} ; 1 \%\right)$} & \multicolumn{2}{|c|}{$\operatorname{Rough}\left(\mathrm{D}_{q, p}^{*, c} ; 5 \%\right)$} & \multicolumn{2}{|c|}{$\operatorname{Good}\left(\mathrm{D}_{q, p}^{*, c} ; 1 \%\right)$} \\
\hline $\begin{array}{l}\text { Shape } \\
(*)\end{array}$ & $\begin{array}{l}\text { Field } \\
(p)\end{array}$ & $\begin{array}{l}\text { Axis } \\
(q)\end{array}$ & $\begin{array}{l}\text { Position } \\
(>)\end{array}$ & $\begin{array}{l}\text { Values }\left(\times 10^{-2}\right) \\
\& \text { error } \mathrm{D}_{q, p}^{*, e}\end{array}$ & $\begin{array}{l}\text { Position } \\
(>)\end{array}$ & $\begin{array}{l}\text { Values }\left(\times 10^{-2}\right) \\
\& \text { error } \mathrm{D}_{q, p}^{* e,}\end{array}$ & $\begin{array}{l}\text { Position } \\
(>)\end{array}$ & $\begin{array}{l}\text { Values }\left(\times 10^{-3}\right) \\
\& \text { error } \mathrm{D}_{q, p}^{*, c}\end{array}$ & $\begin{array}{l}\text { Position } \\
\text { (>) }\end{array}$ & $\begin{array}{l}\text { Values }\left(\times 10^{-3}\right) \\
\& \text { error } \mathrm{D}_{q, p}^{*, c}\end{array}$ \\
\hline \multirow{2}{*}{$\begin{array}{l}\text { A } \\
(0.901)\end{array}$} & $\begin{array}{l}\Gamma_{2} \\
(p=2)\end{array}$ & $\begin{array}{l}x_{1} \\
(\theta=0)\end{array}$ & \multirow{4}{*}{\multicolumn{4}{|c|}{-}} & 2.269 & $\begin{array}{l}-0.9170 \\
4.9 \%\end{array}$ & 3.474 & $\begin{array}{l}-4.105 \\
0.89 \%\end{array}$ \\
\hline & $\begin{array}{l}\Gamma_{4} \\
(p=4)\end{array}$ & $\begin{array}{l}x_{1} \\
(\theta=0)\end{array}$ & & & & & 3.368 & $\begin{array}{l}-0.1822 \\
4.8 \%\end{array}$ & 5.672 & $\begin{array}{l}-0.7330 \\
0.99 \%\end{array}$ \\
\hline \multirow{2}{*}{$\begin{array}{l}\mathrm{S} \\
(0.886)\end{array}$} & $\begin{array}{l}\Gamma_{2} \\
(p=2)\end{array}$ & $\begin{array}{l}x_{1} \\
(\theta=0)\end{array}$ & & & & & 1.967 & $\begin{array}{l}-1.226 \\
5 \%\end{array}$ & 3.013 & $\begin{array}{l}-5.456 \\
1.1 \%\end{array}$ \\
\hline & $\begin{array}{l}\Gamma_{4} \\
(p=4)\end{array}$ & $\begin{array}{l}x_{1} \\
(\theta=0)\end{array}$ & & & & & 2.783 & $\begin{array}{l}-0.2471 \\
5 \%\end{array}$ & 4.467 & $\begin{array}{l}-1.152 \\
0.95 \%\end{array}$ \\
\hline \multicolumn{3}{|c|}{ Summary } & & & & & \multicolumn{2}{|l|}{$>4$} & \multicolumn{2}{|l|}{$>6$} \\
\hline \multirow{4}{*}{$\begin{array}{l}\mathrm{Q} \\
(1.333)\end{array}$} & \multirow{2}{*}{$\begin{array}{l}\Gamma_{2} \\
(p=2)\end{array}$} & $\begin{array}{l}x_{1} \\
(\theta=0)\end{array}$ & 4.112 & $\begin{array}{l}-3.596 \\
4.9 \%\end{array}$ & 6.983 & $\begin{array}{l}-1.097 \\
0.99 \%\end{array}$ & 8.189 & $\begin{array}{l}-7.842 \\
4.9 \%\end{array}$ & 18.08 & $\begin{array}{l}-1.548 \\
0.91 \%\end{array}$ \\
\hline & & $\begin{array}{l}x_{2} \\
\left(\theta=\frac{\pi}{2}\right)\end{array}$ & 2.322 & $\begin{array}{l}5.398 \\
5 \%\end{array}$ & 3.173 & $\begin{array}{l}3.654 \\
0.98 \%\end{array}$ & 7.941 & $\begin{array}{l}7.535 \\
5 \%\end{array}$ & 18.03 & $\begin{array}{l}1.524 \\
0.97 \%\end{array}$ \\
\hline & \multirow{2}{*}{$\begin{array}{l}\Gamma_{4} \\
(p=4)\end{array}$} & $\begin{array}{l}x_{1} \\
(\theta=0)\end{array}$ & 4.857 & $\begin{array}{l}-1.174 \\
4.9 \%\end{array}$ & 8.650 & $\begin{array}{l}-0.346 \\
1.1 \%\end{array}$ & 9.199 & $\begin{array}{l}-3.049 \\
5.1 \%\end{array}$ & 20.00 & $\begin{array}{l}-0.6297 \\
1.1 \%\end{array}$ \\
\hline & & $\begin{array}{l}x_{2} \\
\left(\theta=\frac{\pi}{2}\right)\end{array}$ & 3.829 & $\begin{array}{l}-0.7922 \\
4.9 \%\end{array}$ & 4.750 & $\begin{array}{l}-0.684 \\
1 \%\end{array}$ & 13.15 & $\begin{array}{l}-1.361 \\
5 \%\end{array}$ & 29.64 & $\begin{array}{l}-0.2813 \\
0.67 \%\end{array}$ \\
\hline \multirow{4}{*}{$\begin{array}{l}\mathrm{R} \\
(0.793)\end{array}$} & \multirow{2}{*}{$\begin{array}{l}\Gamma_{2} \\
(p=2)\end{array}$} & $\begin{array}{l}x_{1} \\
(\theta=0)\end{array}$ & 2.925 & $\begin{array}{l}-10.09 \\
5 \%\end{array}$ & 3.456 & $\begin{array}{l}-6.041 \\
1 \%\end{array}$ & 8.880 & $\begin{array}{l}-6.694 \\
5 \%\end{array}$ & 19.50 & $\begin{array}{l}-1.332 \\
1.3 \%\end{array}$ \\
\hline & & $\begin{array}{l}x_{2} \\
\left(\theta=\frac{\pi}{2}\right)\end{array}$ & 2.286 & $\begin{array}{l}5.503 \\
5 \%\end{array}$ & 4.715 & $\begin{array}{l}1.921 \\
0.98 \%\end{array}$ & 8.472 & $\begin{array}{l}6.616 \\
5 \%\end{array}$ & 17.87 & $\begin{array}{l}1.553 \\
1.2 \%\end{array}$ \\
\hline & \multirow{2}{*}{$\begin{array}{l}\Gamma_{4} \\
(p=4)\end{array}$} & $\begin{array}{l}x_{1} \\
(\theta=0)\end{array}$ & 3.244 & $\begin{array}{l}-3.348 \\
4.9 \% \\
\end{array}$ & 4.041 & $\begin{array}{l}-1.910 \\
0.98 \%\end{array}$ & 10.09 & $\begin{array}{l}-2.544 \\
5 \%\end{array}$ & 22.35 & $\begin{array}{l}-0.5039 \\
1 \%\end{array}$ \\
\hline & & $\begin{array}{l}x_{2} \\
\left(\theta=\frac{\pi}{2}\right)\end{array}$ & 4.343 & $\begin{array}{l}-0.7183 \\
5 \%\end{array}$ & 8.118 & $\begin{array}{l}-0.318 \\
1.2 \%\end{array}$ & 14.02 & $\begin{array}{l}-1.195 \\
5.4 \%\end{array}$ & 31.75 & $\begin{array}{l}-0.2452 \\
1 \%\end{array}$ \\
\hline \multicolumn{3}{|c|}{ Summary } & \multicolumn{2}{|l|}{$>5$} & \multicolumn{2}{|l|}{$>9$} & \multicolumn{2}{|l|}{$>15$} & \multicolumn{2}{|l|}{$>32$} \\
\hline
\end{tabular}


(a)

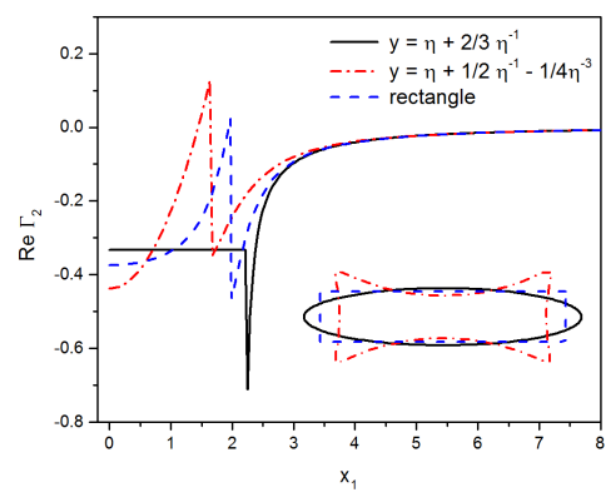

(c)

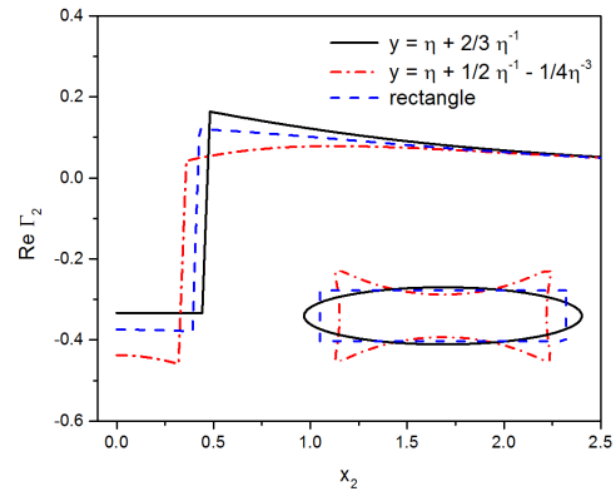

(b)

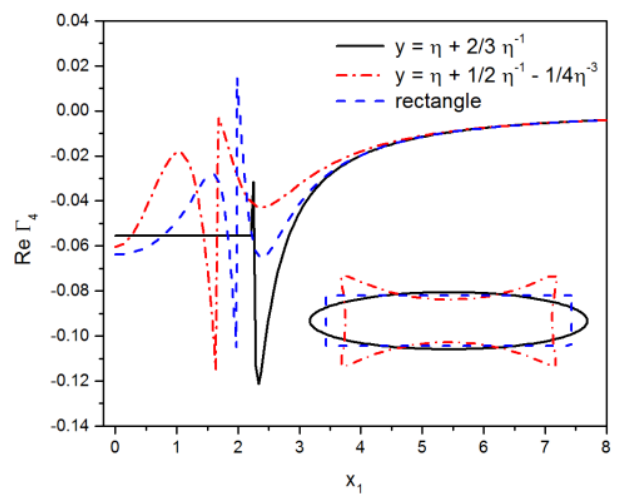

(d)

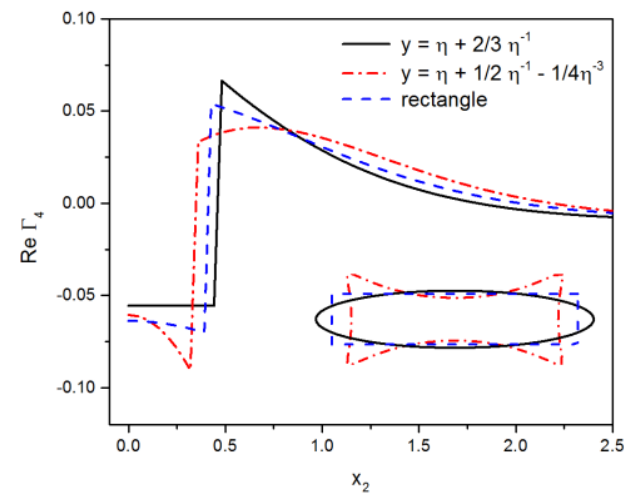

Fig. 5. Variations of $\mathrm{ReG}_{2}$ and $\mathrm{ReG}_{4}$ along the coordinate axes for the three inclusions: elliptical, quasi-parallelogram and rectangular, respectively. The inserted figures in the bottom right corners mark the rescaled boundaries of the inclusions.

\section{Concluding remarks}

In this paper we proposed a novel method to explicitly derive the exterior fields of the inclusion characterized by Laurent polynomials in 2D isotropic elasticity. For the inclusions of particular geometries $((N+1)$-gonal hypocycloids and quasi-parallelograms), the explicit expressions of the two governing functions $\mathrm{G}_{2}$ and $\mathrm{G}_{4}$ are obtained, and the fields of some specific inclusions are compared with those of the benchmarking inclusions (circular, elliptical, normal polygonal) in figures and tables. Results show that the geometry of the inclusion's boundary dominates the fields near the inclusion and greatly alters the uniformity of the interior fields, but slightly affects the exterior fields far away from the inclusion.

The method reported here is elegant and operable, and can be developed in a general routine based on the symbolic computation system. In this sense, it is expectable to obtain more analytical solutions of non-elliptical inclusion problems of this kind. 


\section{Acknowledgments}

This work is funded by National Science Foundation of China (NSFC) under Grant no. 11372124 and Jiangxi Provincial Department of Education under Grant no. GJJ151113, which are gratefully acknowledged. This paper is partly written during the research stay of WNZ at the Université Paris-Est, Laboratoire de Modélisation et Simulation Multi Echelle, as a visiting scholar through QCH and under the financial support of China Scholarship Council (CSC), file no. 201506825048 .

\section{Appendix A. Explicit solutions of interior Eshelby's tensor fields}

\section{for some inclusions described by Laurent polynomials}

Zou et al. (2010) derived the interior fields for some particular inclusions characterized by the Laurent polynomials with finite terms. We list them here for easy reference.

- 3-gonal hypocycloid

$$
\begin{aligned}
2 \mathrm{G}_{2}= & -2 b_{2} \bar{x}, 8 \mathrm{G}_{4}=-6 b_{2}^{2} \bar{x}^{2}+2 b_{2} x \\
& \bullet(N+1) \text {-gonal hypocycloid }\left(N^{3} 3\right) \\
2 \mathrm{G}_{2}= & -N b_{N} \bar{x}^{N-1}, \\
8 \mathrm{G}_{4}= & -(N-1)(N-2)\left(1-n b_{N} \bar{b}_{N}\right) b_{N} \bar{x}^{N-3} \\
& +N(N-1) b_{N} x \bar{x}^{N-2}-N(2 N-1) b_{N}^{2} \bar{x}^{2 N-2}
\end{aligned}
$$

- Quasi-parallelogram

$$
\begin{aligned}
2 \mathrm{G}_{2}= & -b_{1}+3 \bar{b}_{1} b_{3}-3 b_{3} \bar{x}^{2}, \\
8 \mathrm{G}_{4}= & -\left(b_{1}^{2}+9 \bar{b}_{1}^{2} b_{3}^{2}+2 b_{3}-8 b_{1} \bar{b}_{1} b_{3}-6 b_{3}^{2} \bar{b}_{3}\right) \\
& +6\left(b_{3} x \bar{x}-2 b_{1} b_{3} \bar{x}^{2}+6 \bar{b}_{1} b_{3}^{2} \bar{x}^{2}\right)-15 b_{3}^{2} \bar{x}^{4} .
\end{aligned}
$$

\section{Appendix B. Explicit solutions of Eshelby's tensor fields for}

\section{polygonal inclusions}

For an arbitrary polygonal inclusion with $N$ sides, Zou et al. (2010) derived the explicit expressions of its Eshelby's tensor fields $G_{2}$ and $G_{4}$, as

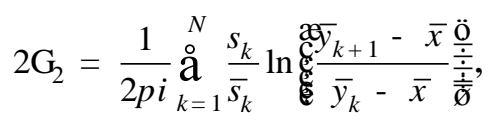




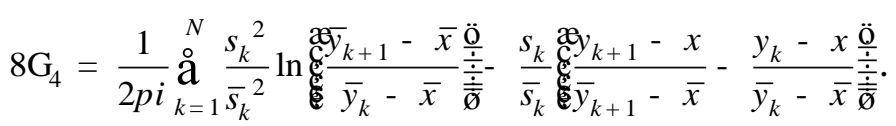

where $x$ is the field point, $y_{k}$ is the corner point of the polygon, and $s_{k}=y_{k+1}-y_{k}$ being the $k$-th side. It is remarked that (B.1) and (B.2) are applicable for both the interior and exterior field points of the inclusion.

\section{References}

Asaro, R., Lubarda, V., 2006. Mechanics of Solids and Materials. Cambridge University Press, Cambridge.

Eshelby, J.D., 1957. The determination of the elastic field of an ellipsoidal inclusion, and related problems. Proc. R. Soc. London, A 241, 376-396.

Eshelby, J.D., 1959. The elastic field outside an ellipsoidal inclusion. Proc. R. Soc. London, A 252, 561-569.

Eshelby, J.D., 1961. Elastic inclusions and inhomogeneities, in: Sneddon, N., Hill, R. (Eds.), Progress in Solid Mechanics. North-Holland, Amsterdam, pp. 89-140.

Henrici, P., 1986. Applied and Computational Complex Analysis. John Wiley \& Sons, New York.

Jin, X., Keer, L.M., Wang, Q., 2011. A closed-form solution for the Eshelby tensor and the elastic field outside an elliptic cylindrical inclusion. J. Appl. Mech. 78, 031009.

Ju, J.W., Sun, L.Z., 1999. A novel formulation for the exterior-point Eshelby's tensor of an ellipsoidal inclusion. J. Appl. Mech. 66, 570-574.

Kim, B.R., Lee, H.K., 2010. Closed form solution of the exterior-point Eshelby tensor for an elliptic cylindrical inclusion. J. Appl. Mech. 77, 024503.

Lubarda, V.A., Markenscoff, X., 1998. On the absence of Eshelby property for non-ellipsoidal inclusions. Int. J. Solids Struct. 35, 3405-3411.

Markenscoff, X., 1998a. Inclusions with constant eigenstress. J. Mech. Phys. Solids 46, 2297-2301.

Markenscoff, X., 1998b. On the shape of the Eshelby inclusions. J. Elast. 49, 163-166.

Miyazawa, T., Aratake, M., Onaka, S., 2012. Superspherical-shape approximation to describe the morphology of small crystalline particles having near-polyhedral shapes with round edges. J. Math. Chem. 50, 249-260.

Mura, T., 1987. Micromechanics of Defects in Solids. Martinus Nijhoff Publishers, Dordrecht.

Mura, T., Cheng, P.C., 1977. The elastic field outside an ellipsoidal inclusion. J. Appl. Mech. 44, 561-569.

Mura, T., Shodja, H.M., Lin, T.Y., Safadi, A., Makkawy, A., 1994. The determination of the elastic field of a pentagonal star shaped inclusion. Bulletin of the Technological University of Istambul 47, 267-280.

Muskhelishvili, N.I., 1977. Some Basic Problems of the Mathematical Theory of Elasticity: 
fundamental equations, plane theory of elasticity, torsion and bending. Springer Science \& Business Media, Dordrecht.

Nemat-Nasser, S., Hori, M., 1999. Micromechanics: Overall Properties of Heterogeneous Materials. Elsevier, Amsterdam.

Nozaki, H., Taya, M., 1997. Elastic fields in a polygon shaped inclusion with uniform eigenstrains. J. Appl. Mech. 64, 495-502.

Nozaki, H., Taya, N., 2001. Elastic fields in a polyhedral inclusion with uniform eigenstrains and related problems. J. Appl. Mech. 68, 441-452.

Onaka, S., 2001. Averaged Eshelby tensor and elastic strain energy of a superspherical inclusion with uniform eigenstrains. Philos. Mag. Lett. 81, 265-272.

Onaka, S., 2005. Strain fields caused by doughnut-like and tubular inclusions with uniform eigenstrains. Mech. Res. Commun. 32, 316-322.

Onaka, S., 2008. Geometrical analysis of near polyhedral shapes with round edges in small crystalline particles or precipitates. J. Mater. Sci. 43, 2680-2685.

Onaka, S., Kobayashi, N., Fujii, T., Kato, M., 2002a. Simplified energy analysis on the equilibrium shape of coherent $\gamma^{\prime}$ precipitates in $\gamma$ matrix with a superspherical shape approximation. Intermetallics 10, 343-346.

Onaka, S., Kobayashi, N., Kato, M., 2002b. Two-dimensional analysis on elastic strain energy due to a uniformly eigenstrained supercircular inclusion in an elastically anisotropic material. Mech. Mater. 34, 117-125.

Rodin, G.J., 1996. Eshelby's inclusion problem for polygons and polyhedra. J. Mech. Phys. Solids 44, 1977-1995.

$\mathrm{Ru}$, C.Q., 1999. Analytic solution for Eshelby's problem of an inclusion of arbitrary shape in a plane or half-plane. J. Appl. Mech. 66, 315-322.

Zheng, Q., Zhao, Z., Du, D., 2006. Irreducible structure, symmetry and average of Eshelby's tensor fields in isotropic elasticity. J. Mech. Phys. Solids 54, 368-383.

Zou, W., He, Q., Huang, M., Zheng, Q., 2010. Eshelby's problem of non-elliptical inclusions. J. Mech. Phys. Solids 58, 346-372.

Zou, W.N., He, Q.C., Zheng, Q.S., 2012. Inclusions in a finite elastic body. Int. J. Solids Struct. 49, 1627-1636. 